\title{
Intra-cluster correlation coefficients in primary care patients with type 2 diabetes and hypertension
}

\author{
Yi Lin Lee 1* $^{*}$, Yvonne Mei Fong Lim², Kian Boon Law ${ }^{1}$ and Sheamini Sivasampu²
}

\begin{abstract}
Introduction: There are few sources of published data on intra-cluster correlation coefficients (ICCS) amongst patients with type 2 diabetes (T2D) and/or hypertension in primary care, particularly in low- and middle-income countries. ICC values are necessary for determining the sample sizes of cluster randomized trials. Hence, we aim to report the ICC values for a range of measures from a cluster-based interventional study conducted in Malaysia.

Method: Baseline data from a large study entitled Evaluation of Enhanced Primary Health Care interventions in public health clinics (EnPHC-EVA: Facility) were used in this analysis. Data from 40 public primary care clinics were collected through retrospective chart reviews and a patient exit survey. We calculated the ICCs for processes of care, clinical outcomes and patient experiences in patients with T2D and/or hypertension using the analysis of variance approach.

Results: Patient experience had the highest ICC values compared to processes of care and clinical outcomes. The ICC values ranged from 0.01 to 0.48 for processes of care. Generally, the ICC values for processes of care for patients with hypertension only are higher than those for T2D patients, with or without hypertension. However, both groups of patients have similar ICCs for antihypertensive medications use. In addition, similar ICC values were observed for clinical outcomes, ranging from 0.01 to 0.09 . For patient experience, the ICCs were between 0.03 (proportion of patients who are willing to recommend the clinic to their friends and family) and 0.25 (for Patient Assessment of Chronic Illness Care item 9, Given a copy of my treatment plan).

Conclusion: The reported ICCs and their respective 95\% confidence intervals for T2D and hypertension will be useful for estimating sample sizes and improving efficiency of cluster trials conducted in the primary care setting, particularly for low- and middle-income countries.
\end{abstract}

Keywords: Intra-cluster correlation, Type 2 diabetes, Hypertension, Primary care, Sample size

\section{Introduction}

Cluster randomized controlled trials (CRCTs) are increasingly being used to study the impact of practice intervention in primary care settings $[1,2]$. It is known that the application of cluster randomization is less demanding to clinical practice and more administratively convenient [3].

\footnotetext{
* Correspondence: yl.yilinlee@gmail.com

${ }^{1}$ Centre for Clinical Trial, Institute for Clinical Research, Ampang Hospital, Ministry of Health, Jalan Mewah Utara, Pandan Mewah, 68000 Ampang, Selangor, Malaysia

Full list of author information is available at the end of the article
}

In CRCTs, subjects are not allocated to intervention independently, but as a group within a cluster. Examples of clusters include communities, schools, clinics and hospitals [4]. Compared to individually randomized trials, CRCTs are not only more complex in design and analysis, but also require more participants to reach equivalent statistical power, as observations in the same cluster tend to be more "alike" $[5,6]$. The condition whereby the effective sample size is less than the total number of participants is referred to as the clustering effect in CRCTs. 
The clustering effect can be determined by calculating the intra-cluster correlation coefficient (ICC), $\rho=\frac{b}{b+w}$, based on measured outcomes, where $b$ is the betweencluster variance and $w$ is the within-cluster variance [5]. Therefore, the ICC can range from 0 to 1 , depending on how varied the measured outcomes would be within a cluster. The sample size of CRCTs often needs to be inflated by the design effect, $\mathrm{DE}=1+(n-1) \rho$, where $n$ is the average number of individuals per cluster. For instance, when there is no clustering effect on a measured outcome, the calculated ICC would be near to zero and the estimated DE may be very close to 1 . A small DE indicates that the clustering effect does not inflate the sample size $[7,8]$.

There have been repeated calls to publish ICCs for future cluster-based interventional studies [7, 9]. Several researchers have published ICCs from large primary care trials conducted in high-income countries [2, 7, 10, 11]. In this paper, we report information on ICCs from a large study conducted at the public primary care clinics in Malaysia, which is an upper middle-income country. Based on the measured variables, we report the ICCs of processes of care, intermediate clinical outcomes and patients' experience of self-management support amongst patients with type 2 diabetes (T2D) with or without hypertension and patients with hypertension only clustered within the selected primary care clinics. The objective of reporting ICCs is to aid sample size estimation for future CRCTs conducted at the primary healthcare level in low- and middle-income countries, as countries in this income category are more similar in the structure and resources of their healthcare systems compared to high-income countries.

\section{Method}

This study used baseline data from a larger study called the Evaluation of Enhanced Primary Health Care (EnPHC) interventions in public health clinics (EnPHC-EVA: Facility). We reported ICC estimates for a range of process and outcome measures for T2D and hypertension based on an ICC reporting framework recommended by a group of researchers and statisticians with experience in CRCTs [12].

\section{The EnPHC interventions and evaluation study}

EnPHC-EVA: Facility was a quasi-experimental controlled study which aimed to assess the effectiveness of an intervention package, known as the EnPHC package, amongst patients with T2D and hypertension in 40 public primary care clinics in Malaysia. Twenty clinics received the interventions, whilst another 20 served as controls. Eligible patients were Malaysians aged 30 years and older who were diagnosed with T2D and/or hypertension. Patients who were pregnant were excluded. Data were collected via retrospective chart review, patient exit survey, healthcare provider survey and an intervention checklist. The effect of the interventions was then analysed using two quasiexperimental analytical methods: the interrupted time series and difference-in-differences approaches. The EnPHC-EVA: Facility study has been completed, and a detailed protocol is currently under journal review.

The clinics involved were public primary healthcare clinics located in the central and southern states of Malaysia, namely Selangor and Johor. These clinics were selected by the study implementers based on the budget and capacity to implement the EnPHC package. In the public primary care setting, there are seven clinic types, type 1 to type 7 [13]. These clinic types are classified by total daily attendances; type 1 is the largest with more than 800 patient visits a day, and type 7 has less than 50 visits daily. The clinics included within this sample were between types 2 to 4 , which represented about $45 \%$ of the public primary clinics in Malaysia based on an unpublished Family Health Development Division report in 2019. The selection criteria for the clinics were at least two medical doctors and 300-800 patient attendances daily. The clinics were then matched in pairs based on the number of medical doctors, number of family medicine specialists, geographical location (urban or rural), annual patient attendance and availability of electronic medical records before being randomly allocated to intervention and control groups.

\section{The EnPHC package included three aspects:}

1. Community engagement. People in the community who resided within the catchment area of the intervention clinics were assigned to appropriate health programs according to their respective cardiovascular risks.

2. Person-centred care bundles. These included cardiovascular risk stratification, assignment to family health teams and task shifting from doctors to other healthcare professionals to improve the continuity and comprehensiveness of chronic care.

3. Integrated care network. Information flow and continuity of care between primary care and other levels of care were improved through standardized referral forms and the role of a care coordinator.

There were four phases of data collection for 31 time points (in months): (1) November 2016 to June 2017 (pre-intervention), (2) August 2017 to June 2019 (postintervention). For the present analysis, only baseline data from November 2016 to June 2017 were included. Clinic visits were sampled separately according to patients' diagnosis of T2D with or without hypertension or hypertension only, because patient registration and appointments were categorized by these groups in the public clinics. Visits were also stratified by the month of their 
visits, because the analysis on intervention effectiveness also assessed changes in processes of care and outcomes with time. Then, a sampling frame for the clinic visits was created accordingly from the patients' register and samples were selected by systematic random sampling, with the random start number generated using Microsoft Excel. Systematic random sampling was used instead of simple random sampling, because it was more straightforward and practical to implement, given the large number of samples by time points that needed to be drawn [14].

The measurements of interest in this study were processes of care, intermediate clinical outcomes and patients' experience. The processes of care and intermediate clinical outcomes were collected from clinic visit records via retrospective chart review using a standardized data extraction form. Patients' experience was assessed using a questionnaire administered by trained researchers to the patients via face-to-face interview.

We reported ICC values for processes of care and therapeutic targets according to the standard of care for T2D and hypertension $[15,16]$. Examples of the processes of care evaluated are at least one HbA1c measurement within 3 months, a foot examination done within 3 months and a lipid measurement within the past 12 months, whilst the intermediate clinical outcomes include the percentage of patients who achieve HbA1c values of $\leq 8 \%$ and the percentage of patients who achieve target blood pressure values of $\leq 135 / 75 \mathrm{mmHg}$. We also reported the mean values of these intermediate outcomes, because some researchers may want to calculate their study sample sizes based on mean differences.

Patients' experience on self-management support was assessed using a short version of the original Patient Assessment of Chronic Illness Care (PACIC) instrument [17]. The questionnaire contained 3 subscales and 11 items [18], as compared to the original PACIC (5 subscales and 20 items). In order to prevent respondent fatigue and after taking into account the low health literacy level amongst the study population, a consensus was reached by an expert group to only adapt the first 11 items, which are from the first 3 subscales of PACIC (patient activation, delivery system design/decision support and goal-setting/tailoring). Each item can be scored by choosing from the options of "None of the time (1)", "A little of the time (2)", "Some of the time (3)", "Most of the time (4)" and "Always (5)". "None of the time" has a score of 1 and "Always" has a score of 5. The score of each subscale can be calculated by averaging the scores of the items within that subscale [17].

We defined the processes of care and intermediate clinical outcomes as objective measures, whilst patientreported experience was considered a subjective measure.
Ethical approval was granted by the Medical Research and Ethics Committee, Ministry of Health Malaysia (study registration number NMRR-17-267-34768).

\section{Data analysis}

Analyses were undertaken using R version 3.6.2. Missing data ranged from $0.2 \%$ to $27.1 \%$, and complete case analysis was performed. The $\mathrm{R}$ scripts are provided in the Appendix.

A standard one-way analysis of variance (ANOVA) was conducted to generate the ICC by using the mean square values. The estimated ICC is given by the formula [19]

$$
\rho=\frac{M S_{\text {between }}-M S_{\text {within }}}{M S_{\text {between }}+(m-1) M S_{\text {within }}}
$$

where $M S$ indicates the mean square values from ANOVA and $m$ is the average cluster size. The $95 \%$ confidence interval (CI) was estimated using Smith's large sample approximation [20, 21], which caters for large and normally distributed data.

For binary variables, we used the ANOVA method with Smith's large sample approximation $[20,21]$ in the ICCbin package [20] to generate the ICC and 95\% CI. Examples of binary variables were whether a process of care such as foot examination was conducted for a diabetes patient and the proportion of patients achieving target values for intermediate clinical outcomes.

For continuous data such as the PACIC score and mean intermediate clinical outcome values such as for $\mathrm{HbA1c}$, ICCs were estimated using the ICC1.CI function from the psychometric package [22]. This function performs a one-way ANOVA fixed effects model for continuous data. In this model, the differences between the clusters, which are the fixed or discrete effects, were estimated [23].

The analyses were conducted separately for patients with T2D (with or without hypertension) and patients with hypertension only, because the processes of care and therapeutic targets for patients with diabetes are different from those who only have hypertension. We reported unadjusted ICC values, because unadjusted values were generally recommended for use in sample size calculation [24]. However, researchers should be aware that published ICCs are estimates only, and they are advised to make statistical adjustment during analysis [24].

\section{Results}

A total of 6722 subjects with T2D (with or without hypertension) and 5014 subjects with hypertension only were included in this study for the reporting of results 
Table 1 Baseline characteristics of study population

\begin{tabular}{|c|c|}
\hline Characteristics & Mean (SD) or \\
\hline \multicolumn{2}{|c|}{$\begin{array}{l}\text { Study population for process of care and clinical outcor } \\
\text { 736) }\end{array}$} \\
\hline Age, years & $60.3(11.3)$ \\
\hline Sex $=$ female & $7323(62.4)$ \\
\hline Body mass index & $27.9(5.8)$ \\
\hline \multicolumn{2}{|l|}{ Body mass index, $\mathrm{kg} / \mathrm{m}^{2}$ [25] } \\
\hline < 18.5 (underweight) & $225(1.9)$ \\
\hline 18.5-22.9 (normal) & $1317(11.2)$ \\
\hline 23-27.4 (overweight) & $3001(25.6)$ \\
\hline$>27.4$ (obese) & $4250(36.2)$ \\
\hline \multicolumn{2}{|l|}{ Ethnicity } \\
\hline Malay & $8071(68.8)$ \\
\hline Chinese & $2456(20.9)$ \\
\hline Indian & $1112(9.5)$ \\
\hline Other & $95(0.8)$ \\
\hline \multicolumn{2}{|l|}{ Morbidity/risk factor } \\
\hline $\mathrm{T} 2 \mathrm{D}$ & $6722(57.3)$ \\
\hline Hypertension & $10,271(87.5)$ \\
\hline Hyperlipidaemia & $4951(42.2)$ \\
\hline \multicolumn{2}{|l|}{ Duration of illness, years } \\
\hline $\mathrm{T} 2 \mathrm{D}$ & $6.5(4.3)$ \\
\hline Hypertension & $7.5(5.1)$ \\
\hline Hyperlipidaemia & $5.0(3.3)$ \\
\hline Target organ damage $=$ Yes & $2591(22.1)$ \\
\hline
\end{tabular}

Study population for patient experience $(n=956)$

$\begin{array}{ll}\text { Age, years } & 59.7(10.9) \\ \text { Sex = female } & 576(60.2) \\ \text { Ethnicity } & \\ \text { Malay } & 664(69.5) \\ \text { Chinese } & 200(20.9) \\ \text { Indian } & 88(9.2) \\ \text { Other } & 4(0.4) \\ \text { Educational level } & \\ \text { No formal/primary/lower secondary } & 659(68.9) \\ \text { Upper secondary } & 239(25.0) \\ \text { Tertiary } & 58(6.1) \\ \text { Morbidity/risk factor } & \\ \text { T2D } & 623(65.2) \\ \text { Hypertension } & 808(84.5) \\ \text { Hyperlipidaemia } & 431(45.1) \\ \text { Duration of illness, years } & \\ \text { T2D } & 8.2(7.0) \\ \text { Hypertension } & 8.4(7.9) \\ \text { Hyperlipidaemia } & 5.2(4.5)\end{array}$

Table 1 Baseline characteristics of study population (Continued)

\begin{tabular}{ll}
\hline Characteristics & Mean (SD) or $\boldsymbol{n}(\%)$ \\
\hline $\begin{array}{l}\text { Clinic characteristics } \boldsymbol{n}=\mathbf{4 0} \\
\text { Geographical location }\end{array}$ \\
$\quad$ Rural & $18(45.0)$ \\
Urban & $22(55.0)$ \\
Daily attendances in 2016 & $282(147)$ \\
\hline
\end{tabular}

$S D$ standard deviation, $T 2 D$ type 2 diabetes

on processes of care and intermediate clinical outcomes. For the patient exit survey, 956 patients with T2D and/ or hypertension were interviewed. The baseline characteristics of the study population are described in Table 1 . The mean age of the study population was 60 years old. The patients were predominantly female $(62 \%)$ and of Malay (69\%) ethnicity. The majority of the patients (82\%) were overweight or obese, and about $42 \%$ of them had hyperlipidaemia. The average cluster size ranged from 141 to 168 for T2D patients with or without hypertension and from 94 to 126 for hypertension-only groups.

ICCs for processes of care, intermediate clinical outcomes and patient experience are presented in Tables 2, 3 and 4 respectively. Between the three categories of measures, the highest ICC was observed for patientreported experience with a median ICC of 0.09 (interquartile range $[\mathrm{IQR}] 0.07,0.13$ ) followed by processes of care with a median of 0.04 (IQR $0.02,0.16$ ) and intermediate clinical outcomes with a median of 0.03 (IQR 0.01, 0.08) (see Fig. 1).

For processes of care, the ICC values ranged from 0.01 to 0.48 . The highest ICC was for measurement of body mass index (BMI, 0.48) and liver function test within 12 months (0.37) in T2D patients with or without hypertension. Overall, the ICCs for common processes of care for patients with hypertension only were higher than those for T2D patients with or without hypertension. However, both groups of patients had similar ICCs (0.01 to 0.04) for antihypertensive medications use. The results also showed low ICC estimates for medication prescribing across all medication groups, for both T2D patients with or without hypertension and hypertension-only patients.

As for clinical outcomes, two variables had the highest ICCs of 0.09. They were the proportion of T2D patients with or without hypertension who achieved the target blood pressure of $\leq 130 / 80 \mathrm{mmHg}$ and the mean diastolic blood pressure of hypertension-only patients. These ICCs for clinical outcomes were mostly similar between both disease groups, ranging from 0.01 to 0.09 . Between binary and continuous data types, such as the proportion of T2D patients with or without hypertension who achieved $\mathrm{HbA} 1 \mathrm{c} \leq 7 \%$ versus the mean value of $\mathrm{HbA1c}$, 
Table 2 Intra-cluster correlation coefficients for processes of care

Processes of care Number, $\boldsymbol{N}$ Average cluster size

$n(\%)$

ICC $\quad 95 \% \mathrm{Cl}$

\section{T2D PATIENTS WITH OR WITHOUT HYPERTENSION $(N=6722)$}

Test/assessment done within a specific interval

Test/assessment done on visit day

FBG

RBG

Blood pressure measurement

\section{At least 1 test/assessment within 3 months}

$\mathrm{HbA} 1 \mathrm{c}$

FBG

Foot examination (pulse, neuropathy, ulcer)

\section{At least 1 test/assessment within 6 months}

Body mass index

At least 1 test/assessment within 12 months

Lipid profile

TC
LDL-C
HDL-C
Triglycerides
Complete lipid profile (TC, LDL-C, HDL-C, triglycerides)
Serum creatinine
Urine albumin
Liver function
Visual acuity examination
Fundus examination
Electrocardiography

\section{Glucose-lowering drugs}

Biguanide (Metformin)

Sulphonylurea

Insulin

Alpha-glucosidase inhibitor (acarbose)

\section{Antihypertensive drugs}

ACEI/ARB

Calcium channel blocker

Beta-blocker

Diuretic

Alpha-blocker

\section{Lipid-lowering drugs}

HMG-CoA reductase inhibitor (statin)

Fibrate

$\begin{array}{ll}6722 & 168 \\ 6722 & 168 \\ 6707 & 168\end{array}$

$6722 \quad 168$

$6722 \quad 168$

6722

$$
168
$$

6696

167

6722

6722

6722

6722

6722

6722

6722

6722

6722

6722

6722

Medication prescribed

$\begin{array}{ll}6722 & 168 \\ 6722 & 168 \\ 6722 & 168 \\ 6722 & 168 \\ 6722 & 168 \\ 6722 & 168 \\ 6722 & 168 \\ 6722 & 168 \\ 6722 & 168 \\ & \\ 6722 & 168 \\ 6722 & 168\end{array}$

$\begin{array}{lll}3053(45.4) & 0.18 & 0.11,0.25 \\ 2685(39.9) & 0.14 & 0.08,0.19 \\ 6574(97.8) & 0.03 & 0.01,0.05 \\ & & \\ 2450(36.4) & 0.09 & 0.05,0.13 \\ 3797(56.5) & 0.16 & 0.09,0.22 \\ 1837(27.3) & 0.17 & 0.10,0.23 \\ & & \\ 2699(40.2) & 0.48 & 0.37,0.59 \\ & & \\ & & \\ 5376(80.0) & 0.03 & 0.01,0.04 \\ 4624(68.8) & 0.16 & 0.10,0.22 \\ 4614(68.6) & 0.16 & 0.10,0.23 \\ 5300(78.8) & 0.02 & 0.01,0.04 \\ 4533(67.4) & 0.16 & 0.10,0.22 \\ 5508(81.9) & 0.03 & 0.01,0.04 \\ 3897(58.0) & 0.20 & 0.13,0.28 \\ 3644(54.2) & 0.37 & 0.26,0.47 \\ 3336(49.6) & 0.21 & 0.13,0.28 \\ 2344(34.9) & 0.13 & 0.08,0.18 \\ 3248(48.3) & 0.22 & 0.14,0.29\end{array}$

$5686(84.6) \quad 0.01$

$0.01,0.02$

$3456(51.4) \quad 0.02 \quad 0.01,0.03$

$1977(29.4) \quad 0.02 \quad 0.01,0.03$

$102(1.5) \quad 0.03 \quad 0.02,0.05$

$4242(63.1) \quad 0.04 \quad 0.02,0.05$

$4056(60.3) \quad 0.02 \quad 0.01,0.03$

$1717(25.5) \quad 0.03 \quad 0.01,0.04$

$1535(22.8) \quad 0.03 \quad 0.01,0.05$

$380(5.7) \quad 0.02 \quad 0.01,0.03$

$5250(78.1) \quad 0.04 \quad 0.02,0.06$

$164(2.4) \quad 0.03 \quad 0.01,0.04$ 
Table 2 Intra-cluster correlation coefficients for processes of care (Continued)

\begin{tabular}{|c|c|c|c|c|c|}
\hline Processes of care & Number, $\boldsymbol{N}$ & Average cluster size & $n(\%)$ & ICC & $95 \% \mathrm{Cl}$ \\
\hline \multicolumn{6}{|c|}{ PATIENTS WITH HYPERTENSION $(N=5014)$} \\
\hline \multicolumn{6}{|c|}{ Test/assessment done within a specific interval } \\
\hline \multicolumn{6}{|c|}{ Test/assessment done on visit day } \\
\hline Blood pressure measurement & 4985 & 125 & 4916 (98.6) & 0.05 & $0.03,0.08$ \\
\hline \multicolumn{6}{|l|}{ At least 1 test/assessment done within 12 months } \\
\hline Blood glucose tests (FBG/RBG/HbA1c) & 5014 & 126 & $3590(71.6)$ & 0.09 & $0.05,0.13$ \\
\hline Serum creatinine & 5014 & 126 & $4005(79.9)$ & 0.06 & $0.03,0.09$ \\
\hline Urine albumin & 5014 & 126 & $2087(41.6)$ & 0.23 & $0.15,0.32$ \\
\hline \multicolumn{6}{|l|}{ Lipid profile } \\
\hline TC & 5014 & 126 & $3919(78.2)$ & 0.05 & $0.03,0.08$ \\
\hline LDL-C & 5014 & 126 & $2954(58.9)$ & 0.22 & $0.14,0.30$ \\
\hline $\mathrm{HDL}-\mathrm{C}$ & 5014 & 126 & $2969(59.2)$ & 0.21 & $0.13,0.29$ \\
\hline Triglycerides & 5014 & 126 & $3894(77.7)$ & 0.05 & $0.03,0.08$ \\
\hline Complete lipid profile (TC, LDL-C, HDL-C, triglycerides) & 5014 & 126 & $2920(58.2)$ & 0.21 & $0.13,0.29$ \\
\hline Electrocardiography & 5014 & 126 & $1802(35.9)$ & 0.28 & $0.18,0.37$ \\
\hline \multicolumn{6}{|c|}{ Medication prescribed } \\
\hline \multicolumn{6}{|l|}{ Antihypertensive drugs } \\
\hline Calcium channel blocker & 5014 & 126 & $3972(79.2)$ & 0.01 & $0.002,0.02$ \\
\hline ACEI/ARB & 5014 & 126 & $2210(44.1)$ & 0.04 & $0.02,0.06$ \\
\hline Beta-blocker & 5014 & 126 & $1382(27.6)$ & 0.02 & $0.01,0.04$ \\
\hline Diuretic & 5014 & 126 & $1054(21.0)$ & 0.02 & $0.01,0.03$ \\
\hline Alpha-blocker & 5014 & 126 & $202(4.0)$ & 0.02 & $0.01,0.03$ \\
\hline \multicolumn{6}{|l|}{ Lipid-lowering drugs } \\
\hline HMG-CoA reductase inhibitor & 5014 & 126 & $3427(68.3)$ & 0.04 & $0.02,0.06$ \\
\hline Fibrate & 5014 & 126 & $63(1.3)$ & 0.01 & $0,0.01$ \\
\hline
\end{tabular}

$A C E I$ angiotensin-converting enzyme inhibitor, $A R B$ angiotensin II receptor blocker, $C l$ confidence interval, FBG fasting blood glucose, $H b A 1 c$ glycated haemoglobin, $H D L-C$ high-density lipoprotein cholesterol, HMG-COA hydroxymethylglutaryl-coenzyme A, ICC intra-cluster correlation coefficient, $L D L-C$ low-density lipoprotein cholesterol, RBG random blood glucose, T2D type 2 diabetes, $T C$ total cholesterol

the ICC values were also similar at around 0.01 . This showed that the variations between clusters were largely similar for intermediate clinical outcomes, whether between different patient groups or data types.

For patient experience, the ICC values were between 0.03 (Proportion of patients who are willing to recommend the clinic to their friends and family) and 0.25 (PACIC item 9, Given a copy of my treatment plan). The mean scores for each item in PACIC were at the lower end of the scale, ranging between 1.5 to 2.8 , except for item 5 (Satisfied that my care was well organized), which had a mean score of 4.2. Both the "Patient activation" and "Delivery system design/Practice design" subscales had the same ICC values of 0.11 . The "Goal setting/Tailoring" subscale had a slightly higher ICC value (0.13) than the other two subscales. This showed that there were slightly more differences between clinics in engaging patients to set their treatment goal as part of their self-management support initiative. The average cluster size for this group of patients was 23.9 (range 23-29).

\section{Discussion}

To the best of our knowledge, this study is amongst the first to report ICCs for T2D and hypertension processes of care and intermediate clinical outcomes in a middleincome country. An analysis of 31 primary care studies found that the context or setting in which the ICC is derived influences the size of the ICC [26]. Since the majority of research works on ICCs for T2D and hypertension care were from high-income countries [2, 11, 27], it was necessary to report those from our setting to determine if factors such as differences in organizational structure and resource limitations within a healthcare setting are determinants of the magnitude of ICCs. In this study, we found 
Table 3 Intra-cluster correlation coefficients for intermediate clinical outcomes

\begin{tabular}{|c|c|c|c|c|c|}
\hline Intermediate clinical outcomes & Number, $\boldsymbol{N}$ & Average cluster size & Mean (SD) or $\boldsymbol{n}(\%)$ & ICC & $95 \% \mathrm{Cl}$ \\
\hline \multicolumn{6}{|c|}{ T2D PATIENTS WITH OR WITHOUT HYPERTENSION $(N=6722)$} \\
\hline $\mathrm{HbA} 1 \mathrm{c}, \%$ & 6208 & 155 & $8.3(2.2)$ & 0.02 & $0.01,0.03$ \\
\hline $\mathrm{HbA} 1 \mathrm{c} \leq 7 \%$ & 6208 & 155 & $2135(34.4)$ & 0.01 & $0.002,0.02$ \\
\hline $\mathrm{HbA} 1 \mathrm{c} \leq 8 \%$ & 6208 & 155 & $3291(53.0)$ & 0.01 & $0.002,0.02$ \\
\hline Systolic BP, mmHga & 6712 & 168 & $137.8(17.1)$ & 0.08 & $0.06,0.13$ \\
\hline Diastolic BP, $\mathrm{mmHg}^{\mathrm{a}}$ & 6712 & 168 & $77.8(9.8)$ & 0.08 & $0.05,0.12$ \\
\hline $\mathrm{BP} \leq 130 / 80 \mathrm{mmHg}$ & 6712 & 168 & $1936(28.8)$ & 0.09 & $0.05,0.13$ \\
\hline $\mathrm{BP} \leq 135 / 75 \mathrm{mmHg}$ & 6712 & 168 & $1757(26.2)$ & 0.06 & $0.03,0.09$ \\
\hline $\mathrm{BP} \leq 140 / 80 \mathrm{mmHg}$ & 6712 & 168 & $2991(44.6)$ & 0.08 & $0.05,0.12$ \\
\hline Serum creatinine $(\mu \mathrm{mol} / \mathrm{L})$ & 6455 & 162 & $88.6(69.7)$ & 0.01 & $0.01,0.02$ \\
\hline TC $(\mathrm{mmol} / \mathrm{L})$ & 6360 & 159 & $5.1(1.2)$ & 0.03 & $0.02,0.06$ \\
\hline LDL-C (mmol/L) & 5624 & 141 & $3.0(1.1)$ & 0.03 & $0.02,0.05$ \\
\hline $\mathrm{LDL}-\mathrm{C} \leq 2.6 \mathrm{mmol} / \mathrm{L}$ & 5624 & 141 & $2191(39.0)$ & 0.02 & $0.01,0.03$ \\
\hline $\mathrm{HDL}-\mathrm{C}(\mathrm{mmol} / \mathrm{L})$ & 5492 & 141 & $1.3(0.4)$ & 0.03 & $0.02,0.05$ \\
\hline Triglycerides (mmol/L) & 6335 & 159 & $1.8(1.2)$ & 0.01 & $0.01,0.03$ \\
\hline \multicolumn{6}{|c|}{ PATIENTS WITH HYPERTENSION $(N=5014)$} \\
\hline Systolic BP, mmHg & 5004 & 126 & $137.8(17.0)$ & 0.08 & $0.05,0.13$ \\
\hline Diastolic BP, mmHg ${ }^{a}$ & 5004 & 126 & $78.7(10.9)$ & 0.09 & $0.06,0.14$ \\
\hline $\mathrm{BP}<140 / 90 \mathrm{mmHg}[15]$ & 5004 & 126 & $2644(52.8)$ & 0.05 & $0.02,0.07$ \\
\hline Serum creatinine $(\mu \mathrm{mol} / \mathrm{L})$ & 4738 & 119 & $83.2(64.6)$ & 0.01 & $0.01,0.03$ \\
\hline $\mathrm{TC}(\mathrm{mmol} / \mathrm{L})$ & 4679 & 117 & $5.3(1.1)$ & 0.03 & $0.02,0.06$ \\
\hline LDL-C (mmol/L) & 3800 & 95 & $3.2(1.0)$ & 0.05 & $0.03,0.09$ \\
\hline $\mathrm{LDL}-\mathrm{C} \leq 2.6 \mathrm{mmol} / \mathrm{L}$ & 3800 & 95 & $1082(28.5)$ & 0.03 & $0.01,0.05$ \\
\hline $\mathrm{HDL}-\mathrm{C}(\mathrm{mmol} / \mathrm{L})$ & 3653 & 94 & $1.4(0.4)$ & 0.03 & $0.01,0.05$ \\
\hline Triglycerides (mmol/L) & 4685 & 118 & $1.5(0.9)$ & 0.01 & $0.004,0.02$ \\
\hline
\end{tabular}

$B P$ blood pressure, $C l$ confidence interval, HbA1c glycated haemoglobin, $H D L-C$ high-density lipoprotein cholesterol, ICC intra-cluster correlation coefficient, $L D L-C$ low-density lipoprotein cholesterol, SD standard deviation, T2D type 2 diabetes, TC total cholesterol

${ }^{a}$ Systolic BP and diastolic BP values are the average of two readings

that estimates for process and intermediate outcome indicators were broadly similar to those reported for highincome countries, by Singh et al. and Littenberg et al. for diabetes and hypertension, except for HbA1c and blood pressure readings $[7,11]$.

We have reported confidence intervals (CIs) together with the ICC estimates, which are useful for investigators who want to perform sensitivity analyses during sample size estimation [26]. Measures of precision are recommended to be included when reporting ICCs, and CIs are the preferred mechanism for providing this information [12]. Bell and McKenzie recommended to examine a range of plausible ICC values and then select conservative options when calculating the sample size for cluster trials [24]. We present these results to expand the existing literature on ICCs in primary care, particularly for low- and middle-income settings.

Our findings were consistent with those reported in the previous literature, where ICCs for processes of care were in general higher than for clinical outcomes, albeit to a lesser extent compared to most studies [7, 11, 28]. A high ICC indicates that the implementation of the processes of care is highly associated with practices. This happens because process measures are largely influenced by health providers' behaviours, which are likely to be collectively more similar within the same clinic. We found that ICCs for the processes of care for hypertension-only patients are generally higher than those for the same processes of care in T2D patients with or without hypertension. This suggests that the variation in management of hypertension-only patients differs more between clinics compared to that of patients with T2D. On the other hand, clinical outcomes are based on an interaction of differences in biological characteristics, diet, lifestyle practices, attitudes and behaviour towards health; thereby they will exhibit greater variability within clusters, causing the ICC to move towards zero. Amongst all process of care measures, BMI 
Table 4 Intra-cluster correlation coefficients for patient experience and willingness to recommend

\begin{tabular}{|c|c|c|c|c|c|}
\hline Patient experience measures & Total & $\begin{array}{l}\text { Average cluster } \\
\text { size }\end{array}$ & $\begin{array}{l}\text { Mean (SD) } \\
\text { or } \\
\boldsymbol{n}(\%)\end{array}$ & ICC & $95 \% \mathrm{Cl}$ \\
\hline Patient activation score (1-3) & 956 & 24 & $2.1(1.1)$ & 0.11 & $\begin{array}{l}0.06 \\
0.19\end{array}$ \\
\hline 1. Asked for my ideas when we made a treatment plan & 956 & 24 & $2.1(1.4)$ & 0.09 & $0.05,0.16$ \\
\hline 2. Given choices about treatment to think about & 956 & 24 & $1.8(1.3)$ & 0.09 & $0.05,0.16$ \\
\hline 3. Asked to talk about any problems with my medicines or their effects & 956 & 24 & $2.3(1.5)$ & 0.06 & $0.03,0.12$ \\
\hline Delivery system design/Practice design score (4-6) & 956 & 24 & $2.9(0.9)$ & 0.11 & $\begin{array}{l}0.07 \\
0.20\end{array}$ \\
\hline 4. Given a written list of things I should do to improve my health & 956 & 24 & $1.6(1.2)$ & 0.14 & $0.09,0.23$ \\
\hline 5. Satisfied that my care was well organized & 956 & 24 & $4.2(1.1)$ & 0.08 & $0.05,0.15$ \\
\hline 6. Shown how what I did to take care of my illness influenced my condition & 956 & 24 & $2.8(1.5)$ & 0.06 & $0.03,0.12$ \\
\hline Goal setting/Tailoring score (7-11) & 956 & 24 & $2.1(0.9)$ & 0.13 & $\begin{array}{l}0.08 \\
0.22\end{array}$ \\
\hline 7. Asked to talk about my goals in caring for my illness & 956 & 24 & $2.2(1.5)$ & 0.19 & $0.12,0.29$ \\
\hline 8. Helped to set specific goals to improve my eating or exercise & 956 & 24 & $2.4(1.5)$ & 0.09 & $0.05,0.16$ \\
\hline 9. Given a copy of my treatment plan & 956 & 24 & $2.7(1.8)$ & 0.25 & $0.17,0.36$ \\
\hline $\begin{array}{l}\text { 10. Encouraged to go to a specific group or class to help me cope with my chronic } \\
\text { illness }\end{array}$ & 956 & 24 & $1.5(1.0)$ & 0.07 & $0.03,0.13$ \\
\hline 11. Asked questions either directly or on a survey, about my health habits & 956 & 24 & $1.8(1.3)$ & 0.11 & $0.06,0.18$ \\
\hline $\begin{array}{l}\text { Proportion of patients who are willing to recommend the clinic to their friends and } \\
\text { family }\end{array}$ & 956 & 24 & $857(89.6)$ & 0.03 & $0,0.06$ \\
\hline
\end{tabular}

Cl confidence interval, ICC intra-cluster correlation coefficient, SD standard deviation

measurement, annual electrocardiography and liver function tests had the highest ICC values. This high variability between clinics reflects differences in provider practice styles, documentation and reminders, availability of resources and time constraints between clinics. Similarly, high ICC values were also reported by Gulliford et al. for the recording of annual weight and urine protein measurement for government primary care services in Trinidad and Tobago [29]. The observed lower median ICC for process measures (0.04) compared to that in the study by Singh and colleagues (0.09) [11] is attributable to the more detailed breakdown of medication categories within this study and the fact that medication prescription has low variation between clinics. This low variation in medication prescription is largely due to the use of a common national drug formulary for public clinics and guideline-adherent practices.

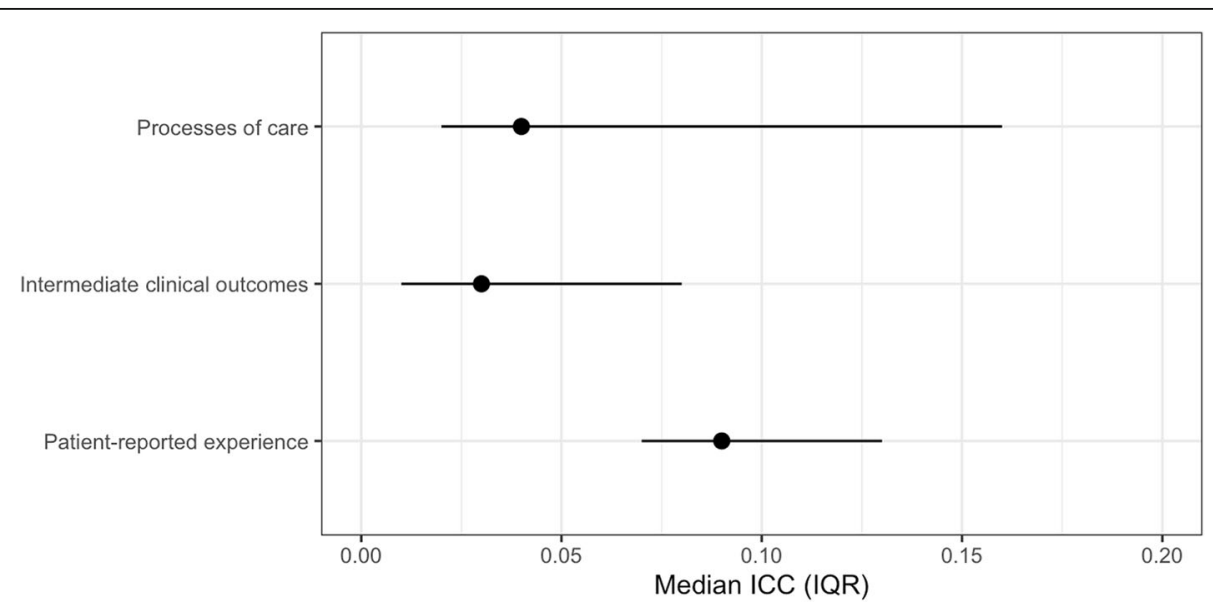

Fig. 1 Median intra-cluster correlation coefficients (ICCS) by processes of care, intermediate clinical outcomes and patient-reported experience, with their respective interquartile ranges (IQRs) 
Prescription of glucose-lowering, antihypertensive and lipid-lowering medications had lower ICCs compared to the laboratory tests and assessments, indicating consistency in pharmacological management of patients with diabetes and hypertension across clinics. We have also found that subjective measures from the patient experience survey had higher ICCs compared to the objective process of care measures. This finding is in agreement with those reported by Campbell et al. [28]. We observed that patient-reported experience had the highest ICC values compared to processes of clinical care and patients' clinical outcomes, suggesting that there was a higher variation between clinic in this aspect, reflecting potential differences in the approach and level of self-management support provided by each clinic's healthcare providers. For example, the high ICC value for PACIC item 9 (Given a copy of my treatment plan) suggests that some of the clinics provide their patients with a document or booklet to increase individual awareness on disease control and treatment, whilst the rest of the clinics are less likely to provide such documents.

There are several limitations in this study. The Malaysian healthcare system consists of two sectors: a governmentled and funded public sector and a private sector which is largely financed by out-of-pocket payments and private insurance [30]. Results from this study are reflective only of public primary care clinics in Malaysia. Data for processes of care and intermediate outcomes were extracted from medical records. Therefore, the results are more likely affected by quality of documentation and the methods of measurement, which may vary between clinics. Lastly, the estimates for low-prevalence processes of care or patient experience should be interpreted with caution due to greater imprecision when assessing measures that occur infrequently [28].

\section{Conclusion}

We have reported ICC estimates and their respective 95\% CIs for a range of measures for T2D and hypertension, which can aid in sample size calculations for cluster randomized controlled trials. This enables more accurate sample size estimation, which allows researchers to calculate realistic recruitment targets whilst reducing the risk of conducting underpowered cluster trials. This is important, particularly in settings where resources are scarce.

\section{Appendix}

These are the $\mathrm{R}$ scripts for generating ICC values by using the ICCbin package and the psychometric package for binary variables and continuous variables respectively. The example shown here for binary variables is the proportion of T2D patients with or without hypertension who have their random blood glucose (RBG) measured on a visit day. The example for continuous variables is the mean of HbA1c of T2D patients with or without hypertension.

Codes for binary variables [20]

library (ICCbin)

iccbin (cid $=$ Site_ID, $y=$ RBGonVisitDate, data $=\mathrm{dm}$,

method $=c($ "aov"), ci.type $=c($ "aov"), alpha $=0.05)$

Details

cid: cluster variable which is Site_ID

$y$ : dependent variable

data: the name of the dataset

method: the method used to compute ICC. In our study,

the ANOVA method was used. The code for ANOVA is "aov"

ci.type: the method used to compute the confidence intervals. We used "aov", which was Smith's large sample approximation

alpha: the significance level for CI. Default value is 0.05. This gives the $95 \% \mathrm{CI}$

Codes for continuous variables [22]

library(psychometric)

ICC1.CI(Lab_HbA1cResult, Site_ID, dm, level = 0.95)

Details

ICC1.CI (dv, iv, data, level $=0.95)$

ICC1.CI: method used to compute the ICC and CI. ICC1.CI computes ICC from one-way ANOVA, and the $C I$ is computed at the desired level using formulae provided by McGraw and Wong [31]

$d v$ : dependent variable

iv: cluster variable which is Site_ID

data: name of the dataset

level: significance level for CI. Default value is 0.95

\section{Abbreviations}

ACEl: Angiotensin-converting enzyme inhibitor; ANOVA: Analysis of variance; ARB: Angiotensin II receptor blocker; BMI: Body mass index; BP: Blood pressure; Cl: Confidence interval; $\mathrm{CRCT}$ : Cluster randomized controlled trial; CVD: Cardiovascular disease; DBP: Diastolic blood pressure; DE: Design effect; ECG: Electrocardiography; EnPHC: Enhanced Primary Health Care; EnPHC-EVA: Facility: Evaluation of Enhanced Primary Health Care (EnPHC) interventions in public health clinics; FBG: Fasting blood glucose; HbA1c: Glycated haemoglobin; HDL-C: High-density lipoprotein cholesterol; HMG-

CoA: Hydroxymethylglutaryl-coenzyme A; ICC: Intra-cluster correlation coefficient; LDL-C: Low-density lipoprotein cholesterol; PACIC: Patient Assessment of Chronic IIIness Care (instrument); SBP: Systolic blood pressure; SD: Standard deviation; T2D: Type 2 diabetes; TC: Total cholesterol;

TG: Triglycerides

\section{Acknowledgements}

We wish to thank the Director-General of Health, Ministry of Health Malaysia for permission to publish the findings. We would also like to thank the patients, health providers and investigators who participated in the EnPHC-EVA: Facility study.

\section{Authors' contributions}

All authors were involved in the conception of this study. YLL was involved in data collection, data analysis and manuscript writing. YMFL collected the data and drafted the manuscript. KBL validated the results and drafted the manuscript. SS was involved in the study design, oversaw the study conduct, acquired the funding and reviewed the manuscript. All authors read and approved the final manuscript. 


\section{Funding}

The EnPHC-EVA: Facility study was supported by a grant from Ministry of Health Malaysia (NMRR-17-267-34768) under the Malaysian Health Systems Research (MHSR) initiative. The opinions, results and conclusions reported in this paper are those of the authors and are independent from the funding source.

\section{Availability of data and materials}

EnPHC-EVA: Facility data were analysed and used for this study. The data are not publicly available due to confidentiality restrictions. However, the data are available from the authors upon reasonable request from the permission of Institute for Clinical Research, Ministry of Health Malaysia. All requests for data should be addressed to the Institute for Clinical Research at contact@crc.gov.my.

\section{Ethics approval and consent to participate}

This study reported ICCs using data from a large observational study, EnPHCEVA: Facility. Ethical approval for EnPHC-EVA: Facility was granted by the Medical Research and Ethics Committee, Ministry of Health Malaysia (NMRR17-267-34768).

\section{Consent for publication}

Not applicable.

\section{Competing interests}

The authors declare that they have no competing interests.

\section{Author details}

${ }^{1}$ Centre for Clinical Trial, Institute for Clinical Research, Ampang Hospital, Ministry of Health, Jalan Mewah Utara, Pandan Mewah, 68000 Ampang Selangor, Malaysia. ${ }^{2}$ Centre for Clinical Outcome Research, Institute for Clinical Research, National Institute of Health, Ministry of Health, Kompleks Institut Kesihatan Negara (NIH), No. 1, Jalan Setia Murni U13/52, Seksyen U13, Setia Alam, 40170 Shah Alam, Selangor, Malaysia.

Received: 24 June 2019 Accepted: 25 April 2020

Published online: 16 June 2020

\section{References}

1. Eldridge SM, Ashby D, Feder GS, Rudnicka AR, Ukoumunne OC. Lessons for cluster randomized trials in the twenty-first century: a systematic review of trials in primary care. Clini Trials. 2004;1:80-90.

2. Martin J, Girling A, Nirantharakumar K, Ryan R, Marshall T, Hemming K. Intracluster and inter-period correlation coefficients for cross-sectional cluster randomised controlled trials for type-2 diabetes in UK primary care. Trials. 2016;17. https://doi.org/10.1186/s13063-016-1532-9.

3. Rutterford C, Taljaard M, Dixon S, Copas A, Eldridge S. Reporting and methodological quality of sample size calculations in cluster randomized trials could be improved: a review. J Clin Epidemiol. 2015;68:716-23.

4. Bland JM. Cluster randomised trials in the medical literature: two bibliometric surveys. BMC Med Res Methodol. 2004:4:21. https://doi.org/10 1186/1471-2288-4-21.

5. Hayes RJ, Bennett S. Simple sample size calculation for cluster-randomized trials. Int J Epidemiol. 1999:28:319-26.

6. Campbell MK, Elbourne DR, Altman DG. CONSORT statement: extension to cluster randomised trials. BMJ. 2004:328:702-8.

7. Littenberg B, MacLean CD. Intra-cluster correlation coefficients in adults with diabetes in primary care practices: the Vermont Diabetes Information System field survey. BMC Med Res Methodol. 2006;6:20

8. Yelland LN, Salter AB, Ryan P, Laurence CO. Adjusted intraclass correlation coefficients for binary data: methods and estimates from a clusterrandomized trial in primary care. Clin Trials. 2011:8:48-58.

9. Ukoumunne OC, Gulliford MC, Chinn S, Sterne JA, Burney PG. Methods for evaluating area-wide and organisation-based interventions in health and health care: a systematic review. Health Technol Assess. 1999:3:iii-92.

10. Parker DR, Evangelou E, Eaton CB. Intraclass correlation coefficients for cluster randomized trials in primary care: the cholesterol education and research trial (CEART). Contemp Clin Trials. 2005;26:260-7.

11. Singh J, Liddy C, Hogg W, Taljaard M. Intracluster correlation coefficients for sample size calculations related to cardiovascular disease prevention and management in primary care practices. BMC Res Notes. 2015;8:89. https://doi.org/10.1186/s13104-015-1042-y.

12. Campbell MK, Grimshaw JM, Elbourne DR. Intracluster correlation coefficients in cluster randomized trials: empirical insights into how should they be reported. BMC Med Res Methodol. 2004;4:9.

13. Bahagian Pembangunan Kesihatan Keluarga (BPKK), Ministry of Health Malaysia. Klasifikasi Klinik Kesihatan. http://fh.moh.gov.my/v3/index.php/infokorporat/pengenalan. Accessed 9 Apr 2020.

14. Bellhouse DR. Systematic sampling methods. In: Wiley StatsRef: statistics reference online. Wiley: Chichester; 2014.

15. Clinical practice guidelines on management of hypertension. 5th ed. Putrajaya: Ministry of Health Malaysia; 2018.

16. Clinical practice guidelines on management of type 2 diabetes mellitus. 5th ed. Putrajaya: Ministry of Health Malaysia; 2015.

17. Glasgow RE, Wagner EH, Schaefer J, Mahoney LD, Reid RJ, Greene SM. Development and validation of the Patient Assessment of Chronic Illness Care (PACIC). Med Care. 2005;43:436-44.

18. Lim MT, Lim YMF, Teh XR, Lee YL, Ismail SA, Sivasampu S. Patient experience on self-management support among primary care patients with diabetes and hypertension. Int J Qual Health Care. 2019:31(7):37-43.

19. Donner A, Birkett N, Buck C. Randomization by cluster. Am J Epidemiol. 1981:114:9.

20. Chakraborty $H$, Hossain A. R package to estimate intracluster correlation coefficient with confidence interval for binary data. Comput Methods Prog Biomed. 2018;155:85-92.

21. Smith CAB. On the estimation of intraclass correlation. Ann Hum Genet 1957:21:363-73

22. Fletcher TD. The psychometric package. R Package; 2006.

23. Armstrong RA, Eperjesi F, Gilmartin B. The application of analysis of variance (ANOVA) to different experimental designs in optometry. Ophthalmic Physiol Opt. 2002;22:248-56

24. Bell ML, McKenzie JE. Designing psycho-oncology randomised trials and cluster randomised trials: variance components and intra-cluster correlation of commonly used psychosocial measures. Psychooncology. 2013;22:1738-47.

25. Clinical practice guidelines on management of obesity. Putrajaya: Ministry of Health Malaysia; 2003.

26. Adams G, Gulliford MC, Ukoumunne OC, Eldridge S, Chinn S, Campbell MJ. Patterns of intra-cluster correlation from primary care research to inform study design and analysis. J Clin Epidemiol. 2004:57:785-94.

27. Knox SA, Chondros P. Observed intra-cluster correlation coefficients in a cluster survey sample of patient encounters in general practice in Australia. BMC Med Res Methodol. 2004:4:30.

28. Campbell MK, Fayers PM, Grimshaw JM. Determinants of the intracluster correlation coefficient in cluster randomized trials: the case of implementation research. Clin Trials. 2005;2:99-107.

29. Gulliford MC, Mahabir D, Ukoumunne OC. Evaluating variations in medical practice between government primary care health centres. J Clin Epidemiol. 2001;54:511-7.

30. World Health Organization. Regional office for the Western Pacific. Malaysia health system review. Manila: WHO Regional Office for the Western Pacific; 2012

31. McGraw KO, Wong SP. Forming inferences about some intraclass correlation coefficients. Psychol Methods. 1996;1:17.

\section{Publisher's Note}

Springer Nature remains neutral with regard to jurisdictional claims in published maps and institutional affiliations.

Ready to submit your research? Choose BMC and benefit from:

- fast, convenient online submission

- thorough peer review by experienced researchers in your field

- rapid publication on acceptance

- support for research data, including large and complex data types

- gold Open Access which fosters wider collaboration and increased citations

- maximum visibility for your research: over $100 \mathrm{M}$ website views per year

At BMC, research is always in progress.

Learn more biomedcentral.com/submission 